\title{
Nonlinear RDT theory of near-wall turbulence
}

\author{
S. Nazarenko ${ }^{\text {a,* }}$, N.K.-R. Kevlahan ${ }^{\text {b }}$, B. Dubrulle ${ }^{\mathrm{c}}$ \\ ${ }^{a}$ Mathematics Institute, University of Warwick, Coventry CV4 7AL, UK \\ ${ }^{\mathrm{b}}$ Department of Mathematics and Statistics, McMaster University, Hamilton, Canada L8S 4K1 \\ ' CNRS URA 2052, L'Orme des Merisiers, 709, 91191 Gif sur Yvette, France
}

Received 12 July 1999; received in revised form 21 October 1999; accepted 21 October 1999

Communicated by A.C. Newell

\begin{abstract}
A WKB method was recently used to extend rapid distortion theory (RDT) to initially inhomogeneous turbulence strained by irrotational mean flows [S.V. Nazarenko, N. Kevlahan, B. Dubrulle, J. Fluid Mech. 390 (1999) 325]. This theory takes into account the feedback of turbulence on the mean flow, and it was used by Nazarenko et al. to explain the effect of strain reduction caused by turbulence observed by Andreotti et al. [B. Andreotti, S. Douady, Y. Couder, in: O. Boratav, A. Eden, A. Erzan (Eds.), Turbulence Modeling and Vortex Dynamics, Proceedings of a Workshop held at Istanbul, Turkey, 2-6 September 1996, pp. 92-108]. In this paper, we develop a similar WKB RDT approach for shear flows. We restrict ourselves to problems where the turbulence is small-scale with respect to the mean flow length-scale and turbulence vorticity is weak compared to the mean shear. We show that the celebrated log-law of the wall exists as an exact analytical solution in our model if the initial turbulence vorticity (debris of the near-wall vortices penetrating into the outer regions) is statistically homogeneous in space and shortly correlated in time. We demonstrate that the main contribution to the shear stress comes from very small turbulent scales which are close to the viscous cut-off and which are elongated in the stream-wise direction (streaks). We also find that anisotropy of the initial turbulent vorticity changes the scaling of the shear stress, but leaves the log-law essentially unchanged. (C2000 Elsevier Science B.V. All rights reserved.
\end{abstract}

Keywords: Nonlinear RDT theory; WKB method; Near-wall turbulence

\section{Introduction}

There has been lively discussion in the literature over several years about whether near-wall turbulence has complete similarity, the classical theory of von Karman [2] and Prandtl [3], or whether it has incomplete similarity, as suggested by Barenblatt et al. [4-6]. The difference between these two cases is important because the former leads to the prediction of a logarithmic mean velocity profile, whereas the latter results in a scaling law characterized by a power-law profile. However, both approaches are based on dimensional analysis, rather than on a theory derived directly from Navier-Stokes equations. They therefore can only be justified by comparison with experimental data (and in some cases model parameters must be found by fitting the data).

\footnotetext{
* Corresponding author.

E-mail address: snazar@maths.warwick.ac.uk (S. Nazarenko).
} 
In an attempt to resolve this controversy, Zagarola et al. [7,8] performed a new experiment in an air pipe under high pressure (called the "Superpipe") which allowed them to reduce the kinematic viscosity by an order of magnitude compared to the classical water pipe experiment of Nikuradze [17]. Based on their data, Zagarola et al. [7,8] argued that there are two overlap regions in the velocity profile: the one closer to the wall has a power-law mean velocity, while the other one follows by the log-law of the wall. Recent papers by Oberlack [9] showed that the underlying symmetries of the Navier-Stokes equations permit power-law, logarithmic and even exponential profiles. George et al. [10] generalized the scaling arguments and obtained a shifted log-law which fits the Superpipe data better than the classical log-law. Meanwhile, Barenblatt et al. [4-6] continue to claim that their scaling power-law fits the experimental data better than the log-law nearly everywhere outside of the viscous sublayer. Currently, direct numerical simulations (DNS) of near-wall turbulence cannot be performed at Reynolds numbers large enough to obtain a clear scaling for the overlap region [11].

It is becoming clear that further development of the theory is severely hindered by insufficient understanding of the relevant physical processes. This lack of understanding stems from the absence of rigorous results on the turbulence dynamics obtained directly from the Navier-Stokes equations. Indeed, even if valid only for special cases, such results could provide a check on the assumptions used in less rigorous (but more general) phenomenological theories. They would identify the physically important quantities to be measured in future experiments, and the physical mechanisms that must be included in future phenomenological models. The derivation of such rigorous results about near-wall turbulence directly from the Navier-Stokes equations is the main goal of the present paper. Our analytical theory has three ingredients: rapid distortion theory (RDT), the Reynolds averaged mean flow equations, and a model for the initial vorticity that provides turbulence forcing.

The first ingredient, RDT, has been a popular and powerful tool for describing shear-flow turbulence since the work of Moffatt [13] who extended the RDT approach of Batchelor and Proudman [14] to the shear-flow geometry. RDT has a long history, but we will only mention here those works that developed approaches similar to that used in the present paper. Hunt [15] generalized RDT to inhomogeneous mean flows and Nazarenko et al. [1] further generalized it to inhomogeneous turbulence using a WKB formalism based on the Gabor transform (GT). Similar WKB approaches have been developed to describe local instabilities by Lifshitz and Hammeri [16].

The second ingredient, the Reynolds averaged equations, is the standard way of describing the dynamics of a mean flow subjected to turbulent stresses. Our aim is to write the averaged turbulent stresses in this equation in terms of the quantities used in RDT, and thus to obtain a closed coupled system of equations linking the mean flow and the turbulence. Such a system was derived in the two-dimensional case by Dyachenko et al. [18] for the $\beta$-plane dynamics, and was used by Dubrulle and Nazarenko [19] to model two-dimensional Euler turbulence. Similar work has been done for the three-dimensional case of nearly irrotational mean flows by Nazarenko et al. [1] who used GTs of the velocity components to describe inhomogeneous turbulence and the turbulence stresses. In the present paper, we will generalize the derivation of [1] to the case of arbitrary rotational mean flows, with shear-flow geometry as a special case. It is interesting that the idea of coupling RDT and the Reynolds averaged mean flow equations was present in the early work of Moffatt [13]. He derived equations for evolution of a nearly monochromatic wave nonlinearly coupled to a background shear via the averaged Reynolds stress. Moffatt used Fourier transforms with, perhaps, an intuitive understanding that these Fourier transforms depend on a slow coordinate of the inhomogeneous mean flow. Although a WKB technique justifying such approach was not developed in that paper, all the physical results obtained were essentially correct. A similar approach was used by Manin and Nazarenko [20] used to describe nonlinear coupling between Rossby waves and a zonal shear-flow.

However, to describe turbulent shear flows one has to add yet another ingredient to ensure existence of a statistically steady state, the turbulence forcing in the overlap region. This forcing is provided by an initial vorticity that penetrates into the overlap region in the form of debris from coherent vortices generated in the viscous sublayer. The view that near-wall turbulence is a linear driven system was introduced by Landahl [21] who also argued that turbulence 
forcing is produced by vorticity bursts originating in the viscous sublayer and which are intermittent in space and time. We think that intermittence is important in this case because only nonlinear intermittent vortex structures can move far from the wall, whereas, according to RDT, a weaker background vorticity is only transported by the mean flow. Because the viscous sublayer is very thin compared to the overlap region these coherent structures have a scale which is smaller than the characteristic scale of the mean flow in the overlap region. Also, it is natural to think that the initial vorticity debris is weak far from the viscous sublayer because it is diluted in a large volume of fluid. Thus, one can assume that the turbulence forcing is weak and small-scale, which allows one to use the RDT approach and the scale separation technique. We will give more detail on the restrictions on the amplitude and scale of the turbulence later. As we will see, the structure of the near-wall turbulence strongly depends on properties of the turbulent forcing such as its statistical spatial uniformity and the decay rate of its correlations. Therefore, the initial vorticity is an important quantity to be studied in future experiments and to be included in more general phenomenological descriptions [22].

The ideas outlined above can be most easily illustrated using two-dimensional shear-flow turbulence as an example [23]. This example is unrealistic for real applications but its algebra is much simpler than that of three-dimensional RDT theory and it can be used as a quick introduction to the theory developed in the present paper.

\section{Dynamics of turbulence}

Let us consider a velocity field in three-dimensional space that consists of a strong large-scale mean component $\boldsymbol{U}$ and weak small-scale fluctuations $\boldsymbol{u}$

$$
\begin{aligned}
& \text { velocity }=\boldsymbol{U}(\boldsymbol{x}, t)+\boldsymbol{u}(\boldsymbol{x}, t), \\
& \boldsymbol{U}=\langle\text { velocity }\rangle, \\
& \langle\boldsymbol{u}\rangle=0, \\
& L \sim U /|\nabla U| \gg u /|\nabla u| \sim l, \\
& U / L \gg u / l .
\end{aligned}
$$

Let us define the Gabor transform (GT)

$$
\hat{\boldsymbol{u}}(\boldsymbol{x}, \boldsymbol{k}, t)=\int f\left(\epsilon^{*}\left|\boldsymbol{x}-\boldsymbol{x}_{0}\right|\right) \mathrm{e}^{\mathrm{i} \boldsymbol{k} \cdot\left(\boldsymbol{x}-\boldsymbol{x}_{0}\right)} \boldsymbol{u}\left(\boldsymbol{x}_{0}, t\right) \mathrm{d} \boldsymbol{x}_{0},
$$

where $1 \gg \epsilon^{*} \gg \epsilon$ and $f(x)$ is a function which decreases rapidly at infinity, e.g. $\exp \left(-x^{2}\right)$. Averaging $\langle\cdot\rangle$ is performed over the statistics of a random force which will be introduced below.

One can think of the GT as a local Fourier transform taken in a box centered at $x$ and having a size which is intermediate between $L$ and $l$. The following properties of the GT will be important for our derivation.

1. The GT commutes with the time and space derivatives, $\partial_{t}$ and $\nabla$. Commutativity with $\partial_{t}$ is obvious. Note that the GT commutes with $\nabla$ only for distances from the boundaries which are larger than the support of function $f$.

2. The condition of scale separation (4) implies that the GT of small-scale fields (e.g. $\hat{\boldsymbol{u}}(\boldsymbol{x}, \boldsymbol{k}, t)$ is finite only for $k \sim 2 \pi / l \gg 2 \pi / L)$.

3. The GT of large-scale fields (e.g. of $\boldsymbol{u}(\boldsymbol{x}, t))$ is exponentially small in $\epsilon^{*}$ for $k \sim 2 \pi / l$. 
4. The rule for taking the GT of derivatives is similar to the one for Fourier transforms in that

$$
\mathrm{GT}(\nabla a)=\mathrm{i} k \hat{a}+\mathrm{O}\left(\epsilon^{*}\right)
$$

where $a$ is a small-scale quantity.

5. The inverse GT is simply an integration over all wavenumbers, e.g.

$$
u(x, t)=\frac{1}{f(0)} \int \hat{\boldsymbol{u}}(\boldsymbol{x}, \boldsymbol{k}, t) \frac{\mathrm{d} \boldsymbol{k}}{(2 \pi)^{3}} .
$$

Let us substitute (1) into the Navier-Stokes equation,

$$
\partial_{t} \boldsymbol{U}+\partial_{t} \boldsymbol{u}+(\boldsymbol{U} \cdot \nabla) \boldsymbol{U}+(\boldsymbol{U} \cdot \nabla) \boldsymbol{u}+(\boldsymbol{u} \cdot \nabla) \boldsymbol{U}+(\boldsymbol{u} \cdot \nabla) \boldsymbol{u}=-\nabla p+\sigma+v \nabla^{2} \boldsymbol{U}+v \nabla^{2} \boldsymbol{u} .
$$

Here $\sigma=\sigma(\boldsymbol{x}, t)$ describes an external random forcing. In the case of near-wall turbulence, this term models the vorticity seeding the outer layers due to the inner-layer vortex structures which break and penetrate into the outer layers and serve as initial turbulence material. We now apply the GT to the above equation with $k \sim 2 \pi / l \sim$ $1 \gg 2 \pi / L \sim \epsilon$ and only retain terms up to first power in $\epsilon$ and $\epsilon^{*}$ (we chose $\epsilon^{*}$ such that $\epsilon^{*} \gg \epsilon \gg\left(\epsilon^{*}\right)^{2}$ ). All large-scale terms (the first and the third ones on the LHS and the third one on the RHS) give no contribution because their GT is exponentially small according to property (3) above. Furthermore, the nonlinear term $(\boldsymbol{u} \cdot \nabla) \boldsymbol{u}$ may be neglected as it is small compared to $(\boldsymbol{u} \cdot \nabla) \boldsymbol{U}$ because of the assumption (5). As a result we have

$$
\partial_{t} \hat{\boldsymbol{u}}+\operatorname{GT}\{(\boldsymbol{U} \cdot \nabla) \boldsymbol{u}\}+(\hat{\boldsymbol{u}} \cdot \nabla) \boldsymbol{U}=-\mathrm{i} k \hat{p}+\hat{\sigma}-v k^{2} \hat{\boldsymbol{u}} .
$$

When finding the GT of $(\boldsymbol{u} \cdot \nabla) \boldsymbol{U}$ we neglected the coordinate dependence of $\partial_{x_{l}} U_{m}$ because this term is already small as $o(\epsilon)$ and any corrections would be of second order. Also, we neglected the order o $(\epsilon)$ correction to the pressure term because, as we will see later, $\hat{p}$ is of order $\epsilon$ itself. We assumed that $\hat{\sigma}=\mathrm{o}(\epsilon)$, which is necessary in order to achieve a stationary state. Finally, we neglected corrections to the viscosity term by assuming that viscosity is of order $\epsilon$ or less. Let us now find

$$
\operatorname{GT}\{(\boldsymbol{U} \cdot \nabla) \boldsymbol{u}\}=\int f\left(\epsilon^{*}\left|\boldsymbol{x}-\boldsymbol{x}_{0}\right|\right) \mathrm{e}^{\mathrm{i} \boldsymbol{k} \cdot\left(\boldsymbol{x}-\boldsymbol{x}_{0}\right)}\left(\boldsymbol{U}\left(\boldsymbol{x}_{0}\right) \cdot \nabla_{x_{0}}\right) \boldsymbol{u}\left(\boldsymbol{x}_{0}\right) \mathrm{d} \boldsymbol{x}_{0} .
$$

Taking into account that $\boldsymbol{U}\left(\boldsymbol{x}_{0}\right)$ varies slowly on distances of order $1 / \epsilon^{*}$ (at which $f$ decays) one can Taylor expand it around $\boldsymbol{x}_{0}=\boldsymbol{x}$. Retaining only the first two terms in the Taylor expansion (which corresponds to retaining only $\mathrm{O}\left(\epsilon^{*}\right)$ and greater), we have

$$
\begin{aligned}
\operatorname{GT}\{(\boldsymbol{u} \cdot \nabla) \boldsymbol{u}\} & =\int f\left(\epsilon^{*}\left|\boldsymbol{x}-\boldsymbol{x}_{0}\right|\right) \mathrm{e}^{\mathrm{i} \boldsymbol{k} \cdot\left(\boldsymbol{x}-\boldsymbol{x}_{0}\right)}\left(\left[\boldsymbol{U}(\boldsymbol{x})+\left(\boldsymbol{x}_{0}-\boldsymbol{x}\right) \cdot \nabla_{x} \boldsymbol{U}(\boldsymbol{x})\right] \cdot \nabla_{x_{0}}\right) \boldsymbol{u}\left(\boldsymbol{x}_{0}\right) \mathrm{d} \boldsymbol{x}_{0} \\
& =(\boldsymbol{U} \cdot \nabla) \hat{\boldsymbol{u}}-\nabla(\boldsymbol{U} \cdot \boldsymbol{k}) \cdot \nabla_{k} \hat{\boldsymbol{u}} .
\end{aligned}
$$

When calculating the second term on the RHS of (12), we took into account that $\left(\boldsymbol{x}_{0}-\boldsymbol{x}\right) \mathrm{e}^{\mathrm{i} k \cdot\left(\boldsymbol{x}-\boldsymbol{x}_{0}\right)}=\mathrm{i} \nabla_{k} \mathrm{e}^{\mathrm{i} \boldsymbol{k} \cdot\left(\boldsymbol{x}-\boldsymbol{x}_{0}\right)}$ and we integrated by parts, neglecting the derivative of $f$ because it is $\epsilon^{*}$ times less than the derivative of $\exp \left[\mathrm{i} k \cdot\left(\boldsymbol{x}-\boldsymbol{x}_{0}\right)\right]$. Thus, (10) becomes

$$
\partial_{t} \hat{\boldsymbol{u}}+(\boldsymbol{U} \cdot \nabla) \hat{\boldsymbol{u}}-\nabla(\boldsymbol{U} \cdot \boldsymbol{k}) \cdot \nabla_{k} \hat{\boldsymbol{u}}+(\hat{\boldsymbol{u}} \cdot \nabla) \boldsymbol{U}=-\mathrm{i} \boldsymbol{k} \hat{p}+\hat{\sigma}-v k^{2} \hat{\boldsymbol{u}} .
$$

We now eliminate $\hat{p}$ by taking into account the incompressibility condition $\nabla \cdot \boldsymbol{u}=0$, which, in terms of the GT, is

$$
\boldsymbol{k} \cdot \hat{\boldsymbol{u}}+\mathrm{O}\left(\epsilon^{*}\right)=0 .
$$

Multiplying (13) by $\boldsymbol{k}$ and taking into account $\boldsymbol{k} \cdot \nabla(\boldsymbol{U} \cdot \boldsymbol{k}) \cdot \nabla_{k} \hat{\boldsymbol{u}}=-\hat{\boldsymbol{u}} \cdot \nabla(\boldsymbol{U} \cdot \boldsymbol{k})$ we have

$$
\hat{p}=\frac{2 \mathrm{i}}{k^{2}} \hat{\boldsymbol{u}} \cdot \nabla(\boldsymbol{U} \cdot \boldsymbol{k})-\frac{\mathrm{i}}{k^{2}}(\boldsymbol{k} \cdot \hat{\boldsymbol{\sigma}}) .
$$


As we see, $\hat{p}=\mathrm{O}\left(\epsilon^{*}\right)$. Substituting $\hat{p}$ into (13) we have

$$
D_{t} \hat{\boldsymbol{u}}+(\hat{\boldsymbol{u}} \cdot \nabla) \boldsymbol{U}=\frac{2 \boldsymbol{k}}{k^{2}} \hat{\boldsymbol{u}} \cdot \nabla(\boldsymbol{U} \cdot \boldsymbol{k})+\hat{\sigma}_{\perp}-v k^{2} \hat{\boldsymbol{u}}
$$

where

$$
\hat{\sigma}_{\perp}=\hat{\sigma}-\frac{k}{k^{2}}(\boldsymbol{k} \cdot \hat{\boldsymbol{\sigma}}),
$$

and

$$
\begin{aligned}
& D_{t}=\partial_{t}+\dot{\boldsymbol{x}} \cdot \nabla+\dot{\boldsymbol{k}} \cdot \nabla_{\boldsymbol{k}}, \\
& \dot{\boldsymbol{x}}=\boldsymbol{U}=\nabla_{k} H, \\
& \dot{\boldsymbol{k}}=-\nabla(\boldsymbol{k} \cdot \boldsymbol{U})=-\nabla H, \\
& H=\boldsymbol{U} \cdot \boldsymbol{k} .
\end{aligned}
$$

Eq. (16) provides an RDT description of turbulence generalized to the case when both the mean flow and the turbulence are inhomogeneous. This equation has the form of a WKB-type transport equation, the characteristics of which (rays) are described by Hamiltonian equations (18) and (19) with Hamiltonian function (20) having the physical meaning of a Doppler shift [1,19]. It is applicable to an arbitrary (slowly varying) mean flow that may contain both vorticity and strain in any proportion. In the special case where the mean flow is irrotational (pure strain) Eq. (16) coincides with the WKB RDT equation derived in [1].

In this paper, we are interested in a shear-flow geometry, so that $\boldsymbol{U}=(U(y, t), 0,0)$. In this case, (16) reduces to

$$
D_{t} \hat{\boldsymbol{u}}-\mathbf{e}_{\mathbf{x}} \Omega \hat{u}_{y}=-\frac{2 \boldsymbol{k}}{k^{2}} \Omega \hat{u}_{y} k_{x}+\hat{\sigma}_{\perp}-v k^{2} \hat{\boldsymbol{u}},
$$

where

$$
D_{t}=\partial_{t}+U \partial_{x}+\Omega k_{x} \partial_{k_{y}},
$$

and $\Omega=-\partial_{y} U$ is the mean flow vorticity.

\section{Dynamics of the mean flow}

In order to derive a mean flow equation one has to filter out the small scales from the Navier-Stokes equations (9) by ensemble averaging over the statistics of the random forcing $\sigma$. Using the component form for convenience, we have

$$
\partial_{t} U_{i}+(\boldsymbol{U} \cdot \nabla) U_{i}+\partial_{x_{l}}\left\langle u_{i} u_{l}\right\rangle=-\partial_{x_{i}} P+v \nabla^{2} U_{i},
$$

where $P=\langle p\rangle$. The nonlinear term $\partial_{x_{l}}\left\langle u_{i} u_{l}\right\rangle$, although small in general, can be the dominant term in the mean flow dynamics. In particular, this is the case for shear flows, where the large-scale self-interaction $(\boldsymbol{U} \cdot \nabla) U_{i}$ is zero because of the parallel geometry, and even small turbulent stresses can lead to significant changes in the mean profile over long times. Eq. (23) is analogous to the equation derived for the large-scale flow by Nazarenko et al. [1] who considered the dynamics of a turbulent spot in an initially irrotational mean flow. The only difference compared to [1] is that in the present paper the averaging is performed over the statistics of a random force, whereas in [1] the large-scale equation was obtained via a space averaging using the filter $f^{2}\left(\epsilon^{*} x\right)$. 
For the shear-flow geometry $\boldsymbol{u}=(U(y), 0,0),(23)$ becomes

$$
\partial_{t} U=-\partial_{y} \tau-\partial_{x} P+v \partial_{y y}^{2} U
$$

where $\tau=\left\langle u_{1} u_{2}\right\rangle$ is the turbulent shear stress. This is the standard equation used for description of turbulent shear flows. The $y$ and $z$ components of the mean flow are not generated by turbulent stresses if the forcing $\sigma$ is statistically symmetric with respect $z \rightarrow-z$, as we will see later.

\section{Solution for $\left\langle u_{i} u_{l}\right\rangle$}

Let us use property (5) of the GT to rewrite the averaged Reynolds stresses $\left\langle u_{i} u_{l}\right\rangle$ as follows:

$$
\left\langle u_{i} u_{l}\right\rangle=\frac{1}{f^{2}(0)(2 \pi)^{6}} \int\left\langle\hat{u}_{i}\left(\boldsymbol{x}, \boldsymbol{k}^{\prime}, t\right) \hat{u}_{l}\left(\boldsymbol{x}, \boldsymbol{k}^{\prime \prime}, t\right)\right\rangle \mathrm{d} \boldsymbol{k}^{\prime} \mathrm{d} \boldsymbol{k}^{\prime \prime} .
$$

Here we have to substitute $\hat{u}_{i}$ found by solving the RDT (16) (21 in case of the shear-flow geometry). Because Eq. (16) is linear, one can write its solution in the following form:

$$
\hat{u}_{i}(\boldsymbol{x}, \boldsymbol{k}, t)=\int g_{i j}\left(\boldsymbol{x}, \boldsymbol{k}, t ; \boldsymbol{x}^{\prime}, \boldsymbol{k}^{\prime}, t^{\prime}\right) \hat{\sigma}_{\perp j}\left(\boldsymbol{x}^{\prime}, \boldsymbol{k}^{\prime}, t^{\prime}\right) \mathrm{d} \boldsymbol{x}^{\prime} \mathrm{d} \boldsymbol{k}^{\prime} \mathrm{d} t^{\prime},
$$

where $g_{i j}$ is the Green's function. Substituting this expression into (25) we have

$$
\begin{aligned}
\left\langle u_{i} u_{l}\right\rangle= & \frac{1}{f^{2}(0)(2 \pi)^{6}} \int g_{i j}\left(\boldsymbol{x}, \boldsymbol{k}^{\prime}, t ; \boldsymbol{x}_{1}, \boldsymbol{k}_{1}, t_{1}\right) g_{l m}\left(\boldsymbol{x}, \boldsymbol{k}^{\prime \prime}, t ; \boldsymbol{x}_{2}, \boldsymbol{k}_{2}, t_{2}\right) \\
& \times\left\langle\hat{\sigma}_{\perp j}\left(\boldsymbol{x}_{1}, \boldsymbol{k}_{1}, t_{1}\right) \hat{\sigma}_{\perp m}\left(\boldsymbol{x}_{2}, \boldsymbol{k}_{2}, t_{2}\right)\right\rangle \mathrm{d} \boldsymbol{x}_{1} \mathrm{~d} \boldsymbol{k}_{1} \mathrm{~d} t_{1} \mathrm{~d} \boldsymbol{x}_{2} \mathrm{~d} \boldsymbol{k}_{2} \mathrm{~d} t_{2} \mathrm{~d} \boldsymbol{k}^{\prime} \mathrm{d} \boldsymbol{k}^{\prime \prime} .
\end{aligned}
$$

Using the definition of the GT we have

$$
\begin{aligned}
\left\langle\hat{\sigma}_{\perp j}\left(\boldsymbol{x}_{1}, \boldsymbol{k}_{1}, t_{1}\right) \hat{\sigma}_{\perp m}\left(\boldsymbol{x}_{2}, \boldsymbol{k}_{2}, t_{2}\right)\right\rangle= & \int f\left(\epsilon^{*}\left|\boldsymbol{x}_{1}-\boldsymbol{x}^{\prime}\right|\right) f\left(\epsilon^{*}\left|\boldsymbol{x}_{2}-\boldsymbol{x}^{\prime \prime}\right|\right) \mathrm{e}^{\mathrm{i} \boldsymbol{k}_{1} \cdot\left(\boldsymbol{x}_{1}-\boldsymbol{x}^{\prime}\right)+\mathrm{i} \boldsymbol{k}_{2} \cdot\left(\boldsymbol{x}_{2}-\boldsymbol{x}^{\prime \prime}\right)} \\
& \times\left\langle\sigma_{\perp j}\left(\boldsymbol{x}^{\prime}, t_{1}\right) \sigma_{\perp m}\left(\boldsymbol{x}^{\prime \prime}, t_{2}\right)\right\rangle \mathrm{d} \boldsymbol{x}^{\prime} \mathrm{d} \boldsymbol{x}^{\prime \prime} \\
= & \int f^{2}\left(\epsilon^{*}\left|\boldsymbol{x}_{1}-\boldsymbol{x}^{\prime}\right|\right) \mathrm{e}^{\mathrm{i} k_{1} \cdot\left(\boldsymbol{x}_{1}-\boldsymbol{x}^{\prime}\right)+\mathrm{i} \boldsymbol{k}_{2} \cdot\left(\boldsymbol{x}_{2}-\boldsymbol{x}^{\prime}-\mathbf{r}\right)} A_{j m}(\mathbf{r}, \xi) \mathrm{d} \boldsymbol{x}^{\prime} \mathrm{d} r \\
= & (2 \pi)^{3} f^{2}\left(\epsilon^{*}\left|\boldsymbol{x}_{1}-\boldsymbol{x}_{2}\right| / 2\right) \delta\left(\boldsymbol{k}_{1}+\boldsymbol{k}_{2}\right) \mathrm{e}^{\mathrm{i} \boldsymbol{k}_{2} \cdot\left(\boldsymbol{x}_{2}-\boldsymbol{x}_{1}\right)} \hat{A}_{j m}\left(\boldsymbol{k}_{2}, \xi\right),
\end{aligned}
$$

where $\xi=t_{2}-t_{1}, r=x^{\prime \prime}-x^{\prime}$ and

$$
A_{j m}(\boldsymbol{r}, \xi)=\left\langle\sigma_{\perp j}\left(\boldsymbol{x}^{\prime}, t_{1}\right) \sigma_{\perp m}\left(\boldsymbol{x}^{\prime \prime}, t_{2}\right)\right\rangle=\left\langle\sigma_{\perp j}(0,0) \sigma_{\perp m}(\boldsymbol{r}, \xi)\right\rangle
$$

is the second correlator of the random forcing, which is assumed to be homogeneous in space and time (later we will also touch upon the case of forcing which is inhomogeneous in space). Further,

$$
\hat{A}_{j m}(\boldsymbol{k}, \xi)=\int A_{j m}(\boldsymbol{r}, \xi) \mathrm{e}^{\mathrm{i} \boldsymbol{k} \cdot \boldsymbol{r}} \mathrm{d} \boldsymbol{r}
$$

is the Fourier transform of the second correlator $A_{j m}$. When deriving (28) we took into account that $A_{j m}(\boldsymbol{r}, \xi)$ decays faster in $\boldsymbol{r}$ than $f$ does (we put $\boldsymbol{x}^{\prime}=\boldsymbol{x}^{\prime \prime}$ in $f$ ). In other words, we assumed that the forcing is small-scale so that $\hat{A}_{j m}(\boldsymbol{k}, \xi)$ is concentrated at $k \gg 2 \pi / L$. We also took into account that $f$ is a slow function compared to the complex exponential and therefore $\delta\left(\boldsymbol{k}_{1}+\boldsymbol{k}_{2}\right)$ appears after integration over $\boldsymbol{x}^{\prime}$. 
Substituting (28) into (27) we have

$$
\begin{aligned}
\left\langle u_{i} u_{l}\right\rangle= & \frac{1}{(2 \pi)^{3} f^{2}(0)} \int f^{2}\left(\epsilon^{*}\left|\boldsymbol{x}_{1}-\boldsymbol{x}_{2}\right| / 2\right) g_{i j}\left(\boldsymbol{x}, \boldsymbol{k}^{\prime}, t ; \boldsymbol{x}_{1}, \boldsymbol{k}_{1}, t_{1}\right) g_{l m}\left(\boldsymbol{x}, \boldsymbol{k}^{\prime \prime}, t ; \boldsymbol{x}_{2}, \boldsymbol{k}_{2}, t_{1}+\xi\right) \mathrm{e}^{\mathrm{i} \boldsymbol{k}_{2} \cdot\left(\boldsymbol{x}_{2}-\boldsymbol{x}_{1}\right)} \\
& \times \hat{A}_{j m}\left(\boldsymbol{k}_{2}, \xi\right) \mathrm{d} \boldsymbol{x}_{1} \mathrm{~d} t_{1} \mathrm{~d} \boldsymbol{x}_{2} \mathrm{~d} \boldsymbol{k}_{2} \mathrm{~d} \xi \mathrm{d} \boldsymbol{k}^{\prime} \mathrm{d} \boldsymbol{k}^{\prime \prime}
\end{aligned}
$$

In case of the shear-flow geometry, we have to find the Green's function of (21), $g_{i j}\left(\boldsymbol{x}, \boldsymbol{k}, t ; \boldsymbol{x}_{1}, \boldsymbol{k}_{1}, t_{1}\right)$, as a solution of the homogeneous equation

$$
\partial_{t} g_{i j}+U \partial_{x} g_{i j}+\Omega k_{1} \partial_{k y} g_{i j}-\delta_{1 i} \Omega g_{i j}=\frac{2 k_{i}}{k^{2}} \Omega g_{2 j} k_{x}-v k^{2} g_{i j}
$$

with initial conditions

$$
\left.g_{i j}\right|_{t=t_{1}}=\delta_{i j} \delta\left(\boldsymbol{x}-\boldsymbol{x}_{1}\right) \delta\left(\boldsymbol{k}-\boldsymbol{k}_{1}\right) .
$$

But solving this problem is similar to finding the classical RDT solution for constant shear of a monochromatic wave with $k=k_{1}$ at $t=t_{1}$. The only difference is that we now consider a localized wavepacket which is advected by the mean flow. Thus, it is easy to see that

$$
\begin{aligned}
g_{i j}\left(\boldsymbol{x}, \boldsymbol{k}, t ; \boldsymbol{x}_{1}, \boldsymbol{k}_{1}, t_{1}\right)= & \kappa_{i j} D \delta\left(x-x_{1}-U \xi_{1}\right) \delta\left(y-y_{1}\right) \delta\left(z-z_{1}\right) \delta\left(k_{x}-k_{1 x}\right) \\
& \times \delta\left(k_{y}-k_{1 y}-\Omega k_{1 x} \xi_{1}\right) \delta\left(k_{z}-k_{1 z}\right),
\end{aligned}
$$

where $\xi_{1}=t-t_{1}, D$ is a viscosity factor,

$$
D=\exp \left[-2 \nu \xi_{1}\left(k_{1}^{2}+\Omega k_{1 x} \xi_{1} k_{1 y}+\Omega^{2} \xi_{1}^{2} k_{1 x}^{2} / 3\right)\right]
$$

and the matrix $\kappa_{i j}$ is the same as for the constant-shear RDT solution [13],

$$
\kappa_{i j}=\left(\begin{array}{ccc}
1 & \frac{k_{1}^{2}}{k_{h}^{2}}\left[\left[\frac{k_{z}^{2}}{k_{x} k_{h}} \theta+\frac{k_{x} k_{y}}{k^{2}}\right]\right] & 0 \\
0 & \frac{k_{1}^{2}}{k^{2}} & 0 \\
0 & \frac{k_{z} k_{1}^{2}}{k_{h}^{3}}\left[\left[\theta+\frac{k_{h} k_{y}}{k^{2}}\right]\right] & 1
\end{array}\right),
$$

where

$$
k_{h}=\sqrt{k_{x}^{2}+k_{z}^{2}}, \quad \theta=\arctan \frac{k_{h}}{k_{y}} .
$$

Here, the double square brackets mean taking the difference of values at $t$ and $t_{1}$, for example $[[\theta]]=\theta(\boldsymbol{k})-\theta\left(\boldsymbol{k}_{1}\right)$. Substituting (34) in (31) and integrating using the delta functions, we have

$$
\begin{aligned}
\left\langle u_{i} u_{l}\right\rangle= & \frac{1}{(2 \pi)^{3} f^{2}(0)} \int_{0}^{\infty} \mathrm{d} \xi_{1} \int_{-\infty}^{\xi_{1}} \mathrm{~d} \xi \int_{R^{3}} \mathrm{~d} \boldsymbol{k}_{1} f^{2}\left(\epsilon^{*}|U \xi| / 2\right) \kappa_{i j}\left(\boldsymbol{k}_{1}, \Omega \xi_{1}\right) \kappa_{l m}\left(-\boldsymbol{k}_{1}, \Omega\left(\xi_{1}-\xi\right)\right) \\
& \times D \mathrm{e}^{-\mathrm{i} k_{1 x} U \xi} \hat{A}_{j m}\left(\boldsymbol{k}_{1}, \xi\right) .
\end{aligned}
$$

Note that $\kappa_{i j}$ depends on the coordinate and time only via combination $\Omega(y) \xi$, a fact that will become important later. In most realistic situations the small-scale forcing correlation $A_{j m}(\boldsymbol{k}, \xi)$ decays in time faster than the characteristic large-scale time $1 / \Omega$. In this case

$$
\left\langle u_{i} u_{l}\right\rangle=\frac{1}{(2 \pi)^{3}} \int_{0}^{\infty} \mathrm{d} \xi_{1} \int_{R^{3}} \mathrm{~d} \boldsymbol{k}_{1} \kappa_{i j}\left(\boldsymbol{k}_{1}, \Omega \xi_{1}\right) \kappa_{l m}\left(-\boldsymbol{k}_{1}, \Omega \xi_{1}\right) D \tilde{A}_{j m}\left(\boldsymbol{k}_{1},-k_{1 x} U\right),
$$


where $\tilde{A}_{j m}(\boldsymbol{k}, w)$ is the Fourier transform of $\left(f^{2}\left(\epsilon^{*}|U \xi| / 2\right) / f^{2}(0)\right) \hat{A}_{j m}(\boldsymbol{k}, \xi)$ with respect to time $\xi$,

$$
\tilde{A}_{j m}(\boldsymbol{k}, w)=\int \frac{f^{2}\left(\epsilon^{*}|U \xi| / 2\right)}{f^{2}(0)} \mathrm{e}^{-\mathrm{i} w \xi} \hat{A}_{j m}(\boldsymbol{k}, \xi) \mathrm{d} \xi .
$$

In the case when the forcing correlation time is smaller than the small-scale time $1 / U k_{x}$ we have

$$
\tilde{A}_{j m}=\int \hat{A}_{j m}(\boldsymbol{k}, \xi) \mathrm{d} \xi
$$

so that $A_{j m}$ is independent of the second (frequency) variable and, therefore, $\tilde{A}_{j m}\left(\boldsymbol{k},-k_{1 x} U\right) \equiv \tilde{A}_{j m}(\boldsymbol{k})$ is independent of $U(y)$. In this case, the turbulent Reynolds stresses $\left\langle u_{i} u_{l}\right\rangle$ depend on the coordinate only via $\Omega$ which, as we shall see later, is crucial for existence of the log solution for $U(y)$ and for some important properties of the spectra. In the following we restrict our attention to this case, leaving other possibilities to another article [22]. Further, we assume the wavenumber dependence of $\tilde{A}_{j m}$ to be of the following standard form:

$$
\tilde{A}_{i j}(\boldsymbol{k})=\frac{3 F(\boldsymbol{k})}{8 \pi}\left(k^{2} \delta_{i j}-k_{i} k_{j}\right),
$$

If, in addition, function $F$ depends only on the absolute value of $k$ then (41) corresponds to an isotropic forcing of turbulence $[13,14]$ (which we are not going to assume).

\section{Turbulent shear stress $\tau$}

We start by calculating $\tau=\left\langle u_{1} u_{2}\right\rangle$, the component of the turbulent Reynolds stress tensor which determines the mean flow dynamics of the shear-flow,

$$
\begin{aligned}
\tau= & \frac{4}{(2 \pi)^{3}} \int_{0}^{\infty} \mathrm{d} \xi \int_{0}^{\infty} \mathrm{d} k_{1 x} \int_{-\infty}^{\infty} \mathrm{d} k_{1 y} \int_{0}^{\infty} \mathrm{d} k_{1 z} \frac{3 F\left(\boldsymbol{k}_{1}\right)}{8 \pi} D \kappa_{22}\left(\boldsymbol{k}_{1}, \Omega \xi\right) \\
& \times\left[\left(k_{1}^{2}-k_{1 x}^{2}\right) \kappa_{12}\left(-\boldsymbol{k}_{1}, \Omega \xi\right)-k_{1 x} k_{1 y} \kappa_{11}\left(-\boldsymbol{k}_{1}, \Omega \xi\right)\right] \\
= & \frac{4}{(2 \pi)^{3}} \int_{0}^{\infty} \mathrm{d} \xi \int_{0}^{\infty} \mathrm{d} k_{1 x} \int_{-\infty}^{\infty} \mathrm{d} k_{1 y} \int_{0}^{\infty} \mathrm{d} k_{1 z} \frac{3 F\left(\boldsymbol{k}_{1}\right)}{8 \pi} \exp \left[-2 \nu \xi\left(k_{1}^{2}+\Omega k_{1 x} \xi k_{1 y}+\Omega^{2} k_{1 x}^{2} \xi^{2} / 3\right)\right] \\
& \times \frac{k_{1}^{2}}{k^{2}}\left(\left(k_{1}^{2}-k_{1 x}^{2}\right) \frac{k_{1}^{2}}{k_{h}^{2}}\left[\left[\frac{k_{z}^{2}}{k_{h} k_{x}} \theta+\frac{k_{x} k_{y}}{k^{2}}\right]\right]-k_{1 x} k_{1 y}\right) .
\end{aligned}
$$

Here, we took into account the symmetry of the integrand with respect to $\boldsymbol{k} \rightarrow-\boldsymbol{k}$ (since $F(-\boldsymbol{k})=F(\boldsymbol{k})$ because the original forcing term $\sigma$ is a real function). Thus, we need only integrate $k_{x}$ from 0 to $\infty$ and double the result. For simplicity we also assumed that $F$ is symmetric with respect to $k_{z} \rightarrow-k_{z}$.

Let us change variables

$$
\left\{\xi, k_{1 x}, k_{1 y}, k_{1 z}\right\} \rightarrow\left\{h=-\Omega k_{1 x} \xi, k_{h}=\sqrt{k_{1 x}^{2}+k_{1 z}^{2}}, k_{y}, s=k_{1 x} / k_{h}\right\} .
$$

Without loss of generality, we assume that $\Omega>0$. Taking into account that the Jacobian associated with this change of variables is $1 / \Omega s \sqrt{1-s^{2}}$, we have

$$
\tau=\frac{1}{\Omega} \int_{0}^{\infty} \mathrm{d} k_{h} \int_{-\infty}^{0} \mathrm{~d} h \int_{-\infty}^{\infty} \mathrm{d} k_{y} \int_{0}^{1} \frac{\mathrm{d} s}{s^{2}} G\left(s, k_{h}, h, k_{y}\right) \mathrm{e}^{-\alpha / s},
$$

where 


$$
\begin{aligned}
G= & \frac{3}{(2 \pi)^{4}} F\left(k_{h} s, k_{y}+h, k_{h} \sqrt{1-s^{2}}\right) \frac{k_{h}^{2}+\left(k_{y}+h\right)^{2}}{\sqrt{1-s^{2}}\left(k_{h}^{2}+k_{y}^{2}\right)}\left\{\frac{k_{h}^{2}+\left(k_{y}+h\right)^{2}}{k_{h}^{2}}\left(k_{h}^{2}\left(1-s^{2}\right)+\left(k_{y}+h\right)^{2}\right)\right. \\
& \times\left[\left(1-s^{2}\right)\left(\arctan \frac{k_{h}}{k_{y}}-\arctan \frac{k_{h}}{k_{y}+h}\right)+k_{h} s^{2}\left(\frac{k_{y}}{k_{h}^{2}+k_{y}^{2}}-\frac{k_{y}+h}{k_{h}^{2}+\left(k_{y}+h\right)^{2}}\right)\right] \\
& \left.-k_{h} s^{2}\left(k_{y}+h\right)\right\},
\end{aligned}
$$

and

$$
\alpha=-\frac{2 v}{\Omega} h\left(k_{h}^{2}+k_{y}^{2}+h k_{y}+h^{2} / 3\right) / k_{h} .
$$

(Note that $\alpha>0$.) One can see that even very small viscosity cannot be neglected here, that is one cannot put $\mathrm{e}^{-\alpha / s}=1$ in the integrand because the factor $1 / s^{2}$ would give rise to a divergence at $s=0$. In order to find the asymptotic expression for $\tau$ for small viscosities, we split the integral over $s$ as follows:

$$
\int_{0}^{1} \frac{\mathrm{d} s}{s^{2}} G(s) \mathrm{e}^{-\alpha / s}=I_{1}+I_{2}+I_{3}
$$

where

$$
\begin{aligned}
& I_{1}=\int_{0}^{1} \frac{\mathrm{d} s}{s^{2}}\left(G(s)-G(0)-s G^{\prime}(0)\right) \mathrm{e}^{-\alpha / s}, \\
& I_{2}=G(0) \int_{0}^{1} \frac{\mathrm{d} s}{s^{2}} \mathrm{e}^{-\alpha / s}=\frac{G(0)}{\alpha}, \\
& I_{3}=G^{\prime}(0) \int_{0}^{1} \frac{\mathrm{d} s}{s} \mathrm{e}^{-\alpha / s}=G^{\prime}(0) \operatorname{Ei}(-\alpha),
\end{aligned}
$$

where $\operatorname{Ei}(x)$ is the exponential-integral function [24] and $G^{\prime}(0)$ denotes the derivative of $G$ with respect to $s$ taken at $s=0$. The expression $\left(G(s)-G(0)-s G^{\prime}(0)\right) / s^{2}$ is not singular at $s=0$ and one can put $\mathrm{e}^{-\alpha / s}=1$ in the integrand of $I_{1}$ in the limit $v \rightarrow 0$,

$$
I_{1}=\int_{0}^{1} \frac{\mathrm{d} s}{s^{2}}\left(G(s)-G(0)-s G^{\prime}(0)\right) .
$$

Also, for $v \rightarrow 0$ one can use the asymptotic expression for $\operatorname{Ei}(\alpha)$ at $\alpha \rightarrow 0$ [24] and write,

$$
I_{3}=G^{\prime}(0)(C+\log \alpha+\mathrm{O}(\alpha)),
$$

where $C$ is Euler's constant. One can see that $I_{2}$ gives the largest contribution to $\tau$. On the other hand, this contribution is independent of $y$ (due to a cancellation of $\Omega$ 's) and therefore does not contribute to the mean flow Eq. (24). Note that this property is very sensitive to the assumptions made about the turbulence forcing, and $I_{2}$ may give the main contribution to the mean flow dynamics if the second correlator of the forcing $\sigma$ decays slowly in time or if it depends on $y$. In our case, the main contribution to $\tau$ comes from the $\log$ term in $I_{3}$ if $G^{\prime}(0) \neq 0$, that is if the correlator of the turbulence forcing is asymmetric in $x$ direction, $\left.\partial_{k x} F\right|_{k_{x}=0} \neq 0$. For the symmetric case $G^{\prime}(0)=\left.\partial_{k x} F\right|_{k_{x}=0}=0$ and the main contribution to $\tau$ comes from $I_{1}$. Summarizing, one can write $\tau$ as a sum of three terms: a constant $\tau_{\mathrm{c}}$ (independent of $y$ ), an asymmetric contribution $\tau_{\mathrm{a}}$, and a symmetric term $\tau_{\mathrm{s}}$. The turbulent shear stress is thus expressed,

$$
\tau=\tau_{\mathrm{c}}+\tau_{\mathrm{a}}+\tau_{\mathrm{s}},
$$


where

$$
\begin{aligned}
& \tau_{\mathrm{c}}=\frac{1}{\Omega} \int_{0}^{\infty} \mathrm{d} k_{h} \int_{-\infty}^{0} \mathrm{~d} h \int_{-\infty}^{\infty} \mathrm{d} k_{y} G(0) / \alpha, \\
& \tau_{\mathrm{a}}=\frac{\lambda_{\mathrm{a}}}{\Omega} \log \frac{\nu k_{*}^{2}}{\Omega}, \\
& \tau_{\mathrm{s}}=\frac{\lambda_{\mathrm{s}}}{\Omega} \\
& \lambda_{\mathrm{a}}=\int_{0}^{\infty} \mathrm{d} k_{h} \int_{-\infty}^{0} \mathrm{~d} h \int_{-\infty}^{\infty} \mathrm{d} k_{y} G^{\prime}(0), \\
& \lambda_{\mathrm{s}}=\int_{0}^{\infty} \mathrm{d} k_{h} \int_{-\infty}^{0} \mathrm{~d} h \int_{-\infty}^{\infty} \mathrm{d} k_{y} \int_{0}^{1} \frac{\mathrm{d} s}{s^{2}}(G(s)-G(0)), \\
& G(0)=F\left(0, k_{y}+h, k_{h}\right) \gamma, \\
& G^{\prime}(0)=\left.\frac{\partial F\left(k_{x}, k_{y}+h, k_{h}\right)}{\partial k_{x}}\right|_{k_{x}=0} k_{h} \gamma, \\
& \gamma=\frac{3}{(2 \pi)^{4}} \frac{\left(k_{h}^{2}+\left(k_{y}+h\right)^{2}\right)^{3}}{\left(k_{h}^{2}+k_{y}^{2}\right)^{2} k_{h}}\left(\arctan \frac{k_{h}}{k_{y}}-\arctan \frac{k_{h}}{k_{y}+h}\right) .
\end{aligned}
$$

Note that when $G^{\prime}(0) \neq 0$, term $\tau_{\mathrm{s}}$ must be ignored because in this case it is of the same order as small corrections to $\tau_{\mathrm{a}}$ which are already neglected in (57). Term $\tau_{\mathrm{s}}$ is only important when $G^{\prime}(0)=0$; thus we put $G^{\prime}(0)=0$ in $(58)$. Note that the sign of $\lambda_{\mathrm{a}}$, and therefore the sign of $\tau_{\mathrm{a}}$, depends on the sign of $G^{\prime}(0)$, i.e. on the way of asymmetry of the turbulence forcing.

\section{Stationary mean flow profile}

\subsection{Qualitative properties of mean flow profile}

The evolution of the mean flow is determined by (24), which is quite general, independent of the actual model (in our case, RDT) used to calculate the turbulent shear stress $\tau$. The RHS of (24) is the negative divergence of the $x$-momentum flux (also called the total shear stress). Taken at the wall, the $y$-component of this flux, i.e. $\tau+v \Omega$, is equal to the friction force per unit area. The friction force is usually written as $u_{*}^{2}$ where $u_{*}$ is called the friction velocity. For stationary mean flow (24) implies

$$
\tau+v \Omega=-(y-H / 2) \partial_{x} P=(1-2 y / H) u_{*}^{2},
$$

where we measure $y$ as the distance from one of the walls and we took into account the fact that the total shear stress must be zero in the center of the channel, at $y=H / 2$. Because we restricted ourselves to the case $\Omega>0$, we have $U<0$ and $\partial_{x} P>0$ (which agrees with $\partial_{x} P=2 u_{*}^{2} / H$ ).

Let us now mention some general properties of the turbulent stress $\tau$ and the mean profile that follow from our model. If $\tau$ is given by (53)-(56) then the vorticity of the stationary mean flow must be a monotonic function of $y$. Indeed, $\Omega(y)$ is not allowed to have a local maximum or minimum because the inverse function $y(\Omega)$ is 


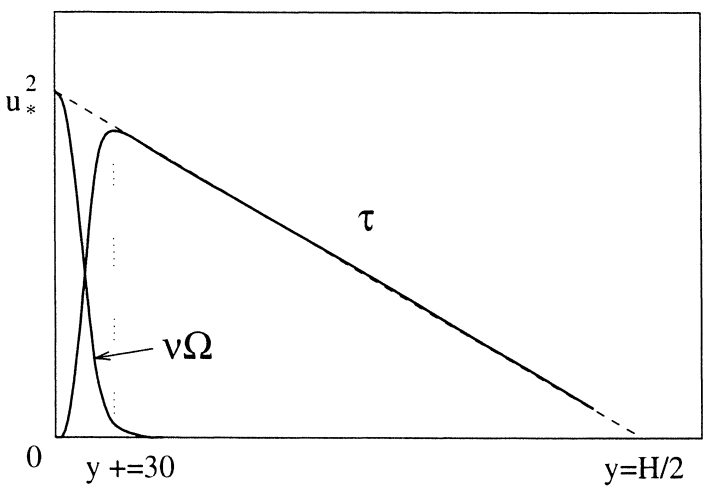

Fig. 1. Sketch of a typical experimentally measured balance between the turbulent stress $\tau$ and viscous shear stress $\nu \Omega$.

uniquely defined via (62). In other words, the stationary mean velocity profile cannot have any inflection points and is, therefore, linearly stable.

The turbulent stress $\tau$ dominates the viscous stress, $\nu \Omega$, everywhere except for a narrow layer near the wall. A typical experimentally measured balance between the turbulent stress $\tau$ and viscous shear stress $\nu \Omega$ is illustrated in Fig. 1. Note that $\tau=0$ at the wall and it has a maximum at $y_{+} \approx 30$, where $y_{+}=y u_{*} / \nu$. The assumptions we made about the weakness of the turbulence forcing and its uniformity are not likely to be valid in the narrow near-wall region $y_{+}<30$ which is dominated by strong coherent vortices; it only makes sense to apply our model to the region $y_{+}>30$. For $y_{+}>30$ the turbulent stress $\tau=0$ tends to a linear function

$$
\tau=(1-2 y / H) u_{*}^{2} .
$$

For $y \ll H$ we have

$$
\tau=\tau_{\mathrm{c}}=u_{*}^{2} .
$$

This follows from the condition that the vorticity profile must be singular at the wall $y=0$ for viscosity to be important when approaching the inner layer, $y \rightarrow 0$. However, when measured in inner variables such a singularity is allowed to be several viscous lengths off the wall, i.e. at $y_{+}=a \sim v / u_{*}$. The constant $a$ can only be found if the inner profile is known (our RDT approach is not applicable there). We will discuss later some consequences of this uncertainty for the mean velocity profile.

Relation (64) represents an integral condition on the level of the turbulence forcing $\sigma$ in the statistically stationary state because such a forcing is the only dynamical quantity that enters into the expression for $\tau_{\mathrm{c}}$. Also, because $\tau_{\mathrm{c}} \propto 1 / \nu, \sigma$ is small for small viscosity, which means RDT is a good description of turbulence in this case. Note that in reality the turbulence forcing is provided by the initial vorticity generated in the viscous sublayer and there is a self-consistent mechanism that adjusts its level to the value necessary to achieve a statistically stationary state. Note that according to (64) $\tau_{\mathrm{c}}$ must be positive; this condition will be an important test for our theory.

Now consider the simplest case, when the turbulence forcing is symmetric so that $\tau_{\mathrm{a}}=0$. In this case the stationary mean profile is to be found from (63) and (56),

$$
\frac{\lambda_{\mathrm{s}}}{\partial_{y} U}=2 y u_{*}^{2} / H,
$$

which gives

$$
U(y)=\frac{\lambda_{\mathrm{s}} H}{2 u_{*}^{2}} \log y+\text { const. }
$$


Note that according to (65) $\lambda_{\mathrm{s}}$ must be negative. This is an important condition for the stationary state to exist, and it will be shown to be satisfied in the next section.

In practical situations the turbulence forcing is likely to be asymmetric. In this case $\tau_{\mathrm{a}} \gg \tau_{\mathrm{s}}$ and we have

$$
\frac{\lambda_{\mathrm{a}}}{\Omega} \log \frac{\nu k_{*}^{2}}{\Omega}=-2 y u_{*}^{2} / H
$$

The constant $\lambda_{\mathrm{a}}$ can be either positive or negative depending on forcing asymmetry, see (57) and Section 6.2. However, according to (67) (taking into account $\Omega>0$ and $\left.\left(v k_{*}^{2}\right) /(\Omega) \ll 1\right)$ a stationary state is only possible if $\lambda_{\mathrm{a}}>0$, so one should expect positive $\lambda_{\mathrm{a}}$ in experiments. Later we will see that $\lambda_{\mathrm{a}}>0$ does correspond to the orientation of the streaks observed in experiments, which means that the real turbulence forcing adjusts itself in such a way that the stationary state is possible.

Let us introduce notation $L=\lambda_{\mathrm{a}} H / 2 v k_{*}^{2} u_{*}^{2}$. Taking into account (64) we see that $L$ is a distance which is of order of the channel width $H$. Solving (67) for $\Omega$ by one iteration we have

$$
\Omega=-v k_{*}^{2} \frac{L}{y} \log \frac{y}{L}
$$

Integrating this equation we have the following mean velocity profile,

$$
U(y)=\frac{1}{2} \nu k_{*}^{2} L \log ^{2} \frac{y}{L}+\text { const. }
$$

As usual, the constant has to be found by matching to the solution in the viscous sublayer, which we cannot do reliably using the present model. In fact, one can show that the relation (69) also gives a log-law dependence in some intermediate range of the coordinates. Indeed, one can rewrite (69) as

$$
U(y)=\frac{1}{2} v k_{*}^{2} L\left(\log ^{2} \frac{y}{L}-\log ^{2} \frac{y_{0}}{L}\right)=-\frac{1}{2} \nu k_{*}^{2} L \log \frac{y y_{0}}{L^{2}} \log \frac{y}{y_{0}},
$$

where constant $y_{0}$ corresponds to the coordinate where the inviscid $\log ^{2}$ profile becomes zero, and is therefore located near the viscous sublayer, $y_{0} \sim v / u_{*}$. For $y_{0} \ll y \ll L$ we have

$$
U(y)=\frac{1}{2} \nu k_{*}^{2} L \log \frac{y_{0}}{L} \log \frac{y}{y_{0}} .
$$

Thus, we see that in the intermediate range $y_{0} \ll y \ll L$ the velocity profile is logarithmic.

As we discussed before, our model is not applicable to the region $y_{+}<30$ which is dominated by strongly nonlinear vortex structures. On the other hand, Eq. (62) can be solved easily for the entire range of $y$ including $y_{+}<30$, and some interesting observations result from matching the resulting profile to the no-slip boundary condition at the wall. For simplicity, let us again consider the symmetric case with $\tau_{\mathrm{a}}=0$. In this case, the solution of (62) satisfying the boundary condition $U(0)=0$ is

$$
U(y)=-\frac{u_{*}^{2} y^{2}}{2 H v}+\frac{y}{2 v} \sqrt{u_{*}^{4} y^{2} / H^{2}+v \lambda_{\mathrm{s}}}+\frac{\lambda_{\mathrm{s}} H}{2 u_{*}^{2}} \log \frac{u_{*}^{2} y / 2 H \nu+2 \sqrt{u_{*}^{4} y^{2} / h^{2}+v \lambda_{\mathrm{s}}}}{2 \sqrt{v \lambda_{\mathrm{s}}}},
$$

which in the limit of small and large distances from the wall gives

$$
\begin{aligned}
& U=2 \sqrt{\frac{\lambda_{\mathrm{s}}}{v}} y \text { for } y^{2} \ll v \lambda_{\mathrm{s}} H^{2} / u_{*}^{4}, \\
& U=\frac{\lambda_{\mathrm{s}} H}{2 u_{*}^{2}} \log \frac{2 u_{*}^{2} y}{H \sqrt{v \lambda_{\mathrm{s}}}} \text { for } y^{2} \ll v \lambda_{\mathrm{s}} H^{2} / u_{*}^{4} .
\end{aligned}
$$


For comparison, let us write the log-law of the wall in its usual form,

$$
U / u_{*}=\frac{1}{\kappa} \log y_{+}+B
$$

Our profile (75) involves the parameter $\lambda_{\mathrm{s}}$ which depends on the properties of the turbulence forcing and is not fixed in our model. However, one may impose a condition that $\lambda_{\mathrm{s}}$ has to be such that the pre-log coefficients in (74) and (75) coincide. Taking (as in the Superpipe experiment [7,8]) the von Karman constant $\kappa=0.44$ and the Reynolds number $R_{*}=u_{*} H / v=851$ we have $B \approx 8.6$. This result is in a reasonable agreement with the experimentally measured value $B \approx 6.5$. Again, such a comparison should be made with caution because our model is not designed to apply to the viscous sublayer. In addition, as we mentioned above, our outer solution can only be found with accuracy up to an arbitrary coordinate offset $a$ of the order of several viscous lengths, which implies that we cannot distinguish between the classical log-law (75) and a shifted log profile

$$
U / u_{*}=\frac{1}{\kappa} \log \left(y_{+}+a\right)+B .
$$

Profile (76) was proposed by George et al. [10] based on a refined scaling argument, and it is quite likely that such a profile describes the near-wall turbulence better than (75).

We would like to emphasize that the logarithmic velocity profile was obtained in this section under several crucial assumptions, one of which being the spatial uniformity of the turbulence forcing. In fact, the existence of the log solution is very sensitive to this particular assumption. Indeed, if the strength of the forcing depends the distance from the wall then the coefficients $\lambda_{\mathrm{s}}$ and $\lambda_{\mathrm{a}}$ will be functions of $y$. For example, if we assume $\lambda_{\mathrm{s}} \propto y^{p}$ then the $\log$ solution (66) will be replaced by a power-law solution. Power-law velocity profiles have been recently suggested as a better description of the near-wall turbulence by Barenblatt et al. [4-6]. Such profiles have also been obtained using a symmetry argument by Oberlack [9]. Our analytical study shows that the realizability of the logarithmic and algebraic profiles depends strongly on the properties of the initial vorticity. Thus, to make a reliable conclusion in favor of the logarithmic or the algebraic profile one has to analyze the initial vorticity, e.g. by using DNS data. In addition to the statistical uniformity of the initial vorticity, these properties include its weakness and the fast decay in time of its second correlator.

\subsection{Evaluation of model constants}

In the previous section we examined qualitatively the shape of the mean flow profile, but did not explicitly evaluate the model constants $\lambda_{\mathrm{s}}, \tau_{\mathrm{c}}, \lambda_{\mathrm{a}}$. These constants depend on the forcing, and in this section we evaluate them for particular forcing models.

We first consider the (rather unphysical) case of symmetric forcing, where the dominant term is $\lambda_{\mathrm{s}}$. In this case we choose the following symmetric forcing:

$$
F(\mathbf{k})=\Gamma \frac{k^{2}}{k_{*}^{2}} \exp \left(-k^{2} / k_{*}^{2}\right) \quad(\Gamma=\text { const }>0),
$$

which behaves like $k^{4}$ as $k \rightarrow 0$ (where $k=|\mathbf{k}|$ ) and decays exponentially for large $k$. Substituting this forcing in (58) gives a multidimensional integral which can only be evaluated analytically in $k_{h}$. The integrals in the other three dimensions have been evaluated numerically using adaptive numerical quadrature to give

$$
\lambda_{\mathrm{s}}=-6.8354 \times 10^{-3} \Gamma k_{*}^{4} .
$$

Note that the constant is negative, as required for existence of the stationary state. The sign of $\lambda_{\mathrm{S}}$ should not depend on the precise form of the forcing (although its magnitude will). 
Next, we consider an asymmetric forcing. This case is more physical, since the forcing in a shear-flow is likely to be asymmetric. We choose a forcing analogous to the symmetric forcing,

$$
F\left(k_{x}, k_{y}, k_{z}\right)=\Gamma \frac{k_{z}^{4}}{k_{*}^{4}} \exp \left\{-\left[\frac{1}{2} a^{2}\left(k_{x}-k_{y}\right)^{2}+\frac{1}{2}\left(k_{x}+k_{y}\right)^{2}+k_{z}^{2}\right] / k_{*}^{2}\right\} \quad(\Gamma=\text { const }>0) .
$$

Note that this forcing corresponds to an ellipse in the $k_{x}-k_{y}$ plane with its major axis at $+45^{\circ}\left(-45^{\circ}\right)$ if $a<1$ $(a>1)$. As $a \rightarrow 0(a \rightarrow \infty)$ the eccentricity of the ellipse increases (the forcing becomes increasingly asymmetric). Note that the $45^{0}$ angle does correspond to the streaks observed in shear layers. In this case the integrals in (57) can be done entirely analytically, giving

$$
\lambda_{\mathrm{a}}=\frac{3^{2}}{2^{5}(2 \pi)^{2}} \frac{\left(1-a^{2}\right)}{\left(1+a^{2}\right)^{4}}\left[16\left(b^{6}+b^{4}+b^{2}+1\right)+5 b\left(b^{4}+1\right)+6 b^{3}\right] \Gamma k_{*}^{4},
$$

where $b^{2}=\frac{1}{2}\left(a^{2}+1\right)$. It is interesting to note that if $a<1$ then $\lambda_{\mathrm{a}}>0$, while if $a>1$ then $\lambda_{\mathrm{a}}<0$. As we mentioned in the previous section, a stationary solution is possible only for negative $\lambda_{\mathrm{a}}$. This selects the type of anisotropy that agrees with experimentally observed orientation of the streaks. This fact not only supports our theory, but it also identifies the mechanism that selects the orientation of the streaks in the statistically stationary state. In the limit of large eccentricities one finds

$$
\begin{aligned}
& \frac{\lambda_{\mathrm{a}}}{\Gamma k_{*}^{4}} \approx-\frac{3^{2}}{2^{4}(2 \pi)^{2}}=-0.01425+\mathrm{O}\left(\frac{1}{a}\right) \quad \text { if } \quad a \gg 1, \\
& \frac{\lambda_{\mathrm{a}}}{\Gamma k_{*}^{4}}=\frac{3^{2}}{2^{5}(2 \pi)^{2}}\left(30+\frac{37}{8} \sqrt{2}\right)=0.26+\mathrm{O}\left(a^{2}\right) \quad \text { if } \quad a \ll 1 .
\end{aligned}
$$

The final constant to be investigated is $\tau_{\mathrm{c}}$, the part of the shear stress independent of $y$. Taking the asymmetric forcing (79) we find that the integral in $k_{h}$ can be done analytically, but the other two must be calculated numerically. When this is done we find that $\tau_{\mathrm{c}}$ is always positive and

$$
\begin{aligned}
& \frac{\tau_{\mathrm{c}} v}{\Gamma k_{*}^{2}} \rightarrow 0 \quad \text { as } a \rightarrow \infty, \\
& \frac{\tau_{\mathrm{c}} v}{\Gamma k_{*}^{2}} \rightarrow 5.3436 \times 10^{-3} \text { as } a \rightarrow 0 .
\end{aligned}
$$

The prediction $\tau_{\mathrm{c}}>0$ agrees with condition (64), which means that the turbulent shear stress must always have the same sign as the viscous stress. This arises because $\tau_{\mathrm{c}} \gg \tau_{\mathrm{s}}, \tau_{\mathrm{a}}$ for small $\nu$.

Our theory also predicts particular scalings of the forcing scale $k_{*}$ and its intensity as a function of the Reynolds number $R_{*}=u_{*} H / v$ and the channel width $H$. Indeed, according to the above formulae and condition (64) we have

$$
\Gamma k_{*}^{2} / v \sim u_{*}^{2} .
$$

On the other hand, comparison of (66) with (75) and taking into account (78) gives

$$
\Gamma k_{*}^{4} H \sim u_{*}^{3} \text {. }
$$

These two relations give the following prediction for the forcing scale

$$
k_{*} H \sim \sqrt{R_{*}} .
$$

Taking into account that $R_{*} \gg 1$ we see that the forcing scale is much less than the channel width which is another justification for the scale separation assumption used in this paper. 
Let us assume that starting vorticity $\omega$ is injected at the rate determined by the characteristic mean flow time $1 / \Omega$ so that $F \sim \Gamma \sim \omega^{2} \Omega$. Taking into account that in the logarithmic layer $\Omega \sim u_{*} / y$ and solving for $\Gamma$ from (85) and (86) we have

$$
\frac{\omega^{2}}{\Omega^{2}} \sim(y / H)^{3} R_{*}^{-2} .
$$

Taking into account that the log region lies at $y<0.1 H$ and that $R_{*} \gg 1$ we see that the initial vorticity is much less than the mean flow vorticity, which justifies the RDT used in this paper.

In this section we have given some indication of the properties a self-consistent forcing should have. This topic will be addressed in more detail in a forthcoming article [22].

\section{Spectra}

The one-dimensional energy spectra $\phi_{i j}\left(k_{x}, y\right)$ are quantities frequently measured in experiment. They are defined in such a way that

$$
\left\langle u_{i} u_{j}\right\rangle=\int \phi_{i j}\left(k_{x}, y\right) \frac{\mathrm{d} k_{x}}{2 \pi} .
$$

Using (38) and (41) we have

$$
\phi_{i l}\left(k_{x}, y\right)=\frac{1}{(2 \pi)^{2}} \int_{0}^{\infty} \mathrm{d} \xi \int_{R^{2}} \mathrm{~d} k_{1 y} \mathrm{~d} k_{1 z} \kappa_{i j}\left(\boldsymbol{k}_{1}, \Omega \xi\right) \kappa_{l m}\left(-\boldsymbol{k}_{1}, \Omega \xi\right) D \frac{3 F\left(\boldsymbol{k}_{1}\right)}{8 \pi}\left(k_{1}^{2} \delta_{j m}-k_{1 j} k_{1 m}\right) .
$$

For $\phi_{11}\left(k_{x}, y\right)$ we have

$$
\begin{aligned}
\phi_{11}= & \frac{1}{(2 \pi)^{2}} \int_{0}^{\infty} \mathrm{d} \xi \int_{R^{2}} \mathrm{~d} k_{1 y} \mathrm{~d} k_{1 z} \frac{3 F\left(\boldsymbol{k}_{1}\right)}{8 \pi}\left[\kappa_{11}^{2}\left(k_{1 y}^{2}+k_{1 z}^{2}\right)-2 \kappa_{12} \kappa_{11} k_{1 x} k_{1 y}+\kappa_{12}^{2} k_{h}^{2}\right] \\
= & \frac{1}{(2 \pi)^{2} k_{x} \Omega} \int_{0}^{\infty} \mathrm{d} \beta \int_{R^{2}} \mathrm{~d} k_{1 y} \mathrm{~d} k_{1 z} \frac{3 F\left(\boldsymbol{k}_{1}\right)}{8 \pi}\left[k_{1 y}^{2}+k_{1 z}^{2}+\frac{k_{1}^{4}}{k_{h}^{2}} A^{2}-2 k_{1 x} k_{1 y} \frac{k_{1}^{2}}{k_{h}^{2}} A\right] \\
& \times \exp \left[-2 \frac{v \beta}{\Omega k_{x}}\left(k_{1}^{2}+\beta k_{1 y}+\beta^{2} / 3\right)\right],
\end{aligned}
$$

where

$$
A=\frac{k_{z}^{2}}{k_{x} k_{h}} \arctan \frac{k_{h}}{k_{1 y}+\beta}+\frac{k_{x} k_{y}}{k^{2}}-\frac{k_{z}^{2}}{k_{x} k_{h}} \arctan \frac{k_{h}}{k_{1 y}}-\frac{k_{x} k_{1 y}}{k_{1}^{2}},
$$

and $\beta=\Omega k_{x} \xi$. Note that the main contribution to this integral comes from $k_{1 y} \sim k_{1 z} \sim k_{*}$, where $k_{*}$ is the characteristic wavenumber of the forcing $F$. We are interested in the limit of small viscosities,

$$
v \ll \Omega k_{x} / k_{*}^{3} .
$$

In this case the main contribution to the integral in $\phi_{11}$ comes from $\beta \gg k_{*}$, so that

$$
\begin{aligned}
\phi_{11}= & \frac{1}{(2 \pi)^{2} k_{x} \Omega} \int_{0}^{\infty} \mathrm{d} \beta \int_{R^{2}} \mathrm{~d} k_{1 y} \mathrm{~d} k_{1 z} \frac{3 F\left(\boldsymbol{k}_{1}\right)}{8 \pi}\left[k_{1 y}^{2}+k_{1 z}^{2}+\frac{k_{1}^{4}}{k_{h}^{2}}\left(\frac{k_{z}^{2}}{k_{x} k_{h}} \arctan \frac{k_{h}}{k_{1 y}}+\frac{k_{x} k_{1 y}}{k_{1}^{2}}\right)^{2}\right. \\
& \left.-2 k_{1 x} k_{1 y} \frac{k_{1}^{2}}{k_{h}^{2}}\left(\frac{k_{z}^{2}}{k_{x} k_{h}} \arctan \frac{k_{h}}{k_{1 y}}+\frac{k_{x} k_{1 y}}{k_{1}^{2}}\right)\right] \exp \left[-\frac{2 v \beta^{3}}{3 \Omega k_{x}}\right] .
\end{aligned}
$$


Integrating over $\beta$ we have

$$
\phi_{11}=\frac{B}{v^{1 / 3} \Omega^{2 / 3}},
$$

where

$$
\begin{aligned}
B= & \frac{3}{4(2 \pi)^{3} k_{x}^{2 / 3}} \int_{0}^{\infty} \mathrm{e}^{-\left(2 p^{3} / 3\right)} \mathrm{d} p \int_{R^{2}} \mathrm{~d} k_{1 y} \mathrm{~d} k_{1 z} F\left(\boldsymbol{k}_{1}\right)\left[k_{1 y}^{2}+k_{1 z}^{2}+\frac{k_{1}^{4}}{k_{h}^{2}}\left(\frac{k_{z}^{2}}{k_{x} k_{h}} \arctan \frac{k_{h}}{k_{1 y}}+\frac{k_{x} k_{1 y}}{k_{1}^{2}}\right)^{2}\right. \\
& \left.-2 k_{1 x} k_{1 y} \frac{k_{1}^{2}}{k_{h}^{2}}\left(\frac{k_{z}^{2}}{k_{x} k_{h}} \arctan \frac{k_{h}}{k_{1 y}}+\frac{k_{x} k_{1 y}}{k_{1}^{2}}\right)\right] .
\end{aligned}
$$

One can see that $\phi_{11}\left(k_{x}, y\right)$ depends on $y$ via $\Omega$ in a universal way. In the log region $\Omega \propto 1 / y$ so that $\phi_{11}\left(k_{x}, y\right) \propto$ $y^{2 / 3}$. The dependence of $\phi_{11}\left(k_{x}, y\right)$ on $k_{x}$ is universal for the wavenumber range $k_{x} \ll k_{*}$, in which case

$$
\phi_{11}=\frac{c_{11}}{v^{1 / 3} \Omega^{2 / 3}} k_{x}^{-8 / 3}
$$

where

$$
c_{11}=\frac{3}{4(2 \pi)^{3}} \int_{0}^{\infty} \mathrm{e}^{-\left(2 p^{3} / 3\right)} \mathrm{d} p \int_{R^{2}} \mathrm{~d} k_{1 y} \mathrm{~d} k_{1 z} F\left(0, k_{1 y}, k_{1 z}\right)\left(k_{1 y}^{2}+k_{1 z}^{2}\right)^{2} \arctan ^{2} \frac{k_{1 z}}{k_{1 y}} .
$$

$\phi_{11}$ is a spectrum that is often measured in experiments. Typically it behaves like $k_{x}^{-1}$ at low wavenumbers and $k_{x}^{-5 / 3}$ for higher $k_{x}[25]$, and both of these scalings are different from the $k_{x}^{-8 / 3}$ obtained here. Note that in the two-dimensional case our theory does predict $\phi_{11} \propto k_{x}^{-1}$, see [23], which may indicate that in reality the turbulence in the $k_{x}^{-1}$ region is effectively two-dimensional. However, one has to be cautious when interpreting our result in terms of the experiments because our theory is not applicable in the range $k_{x} \sim 1 / y$ and below, which is where the experimental data is usually obtained [25]. This is because in the present paper we ignored the turbulence blocking effect which becomes important at $k_{x} \sim 1 / y$ [15]. Inclusion of the turbulence blocking effect is an interesting subject for future research.

The situation with spectra measured in DNS is quite different. The small-scale one-dimensional spectra are indeed measured in DNS, but they turn out to be much steeper than the $k_{x}^{-8 / 3}$ spectrum [11,12]. This difference may be due to numerical dissipation at high $k_{x}$ due to the insufficient Reynolds numbers of current DNS.

To calculate $\phi_{12}\left(k_{x}, y\right)$ we note that in this case the integral converges even if we put $v=0$, so that in the limit of small viscosities dissipation factor $D$ should be ignored. We have

$$
\phi_{12}=\frac{1}{(2 \pi)^{3} \Omega k_{x}} \int_{0}^{\infty} \mathrm{d} \beta \int_{-\infty}^{\infty} \mathrm{d} k_{1 y} \int_{-\infty}^{\infty} \mathrm{d} k_{1 z} \frac{3 F\left(\boldsymbol{k}_{1}\right)}{8 \pi} \frac{k_{1}^{2}}{\left(k_{1 y}+\beta\right)^{2}+k_{h}^{2}}\left[\left(k_{1}^{2}-k_{1 x}^{2}\right) \frac{k_{1}^{2}}{k_{h}^{2}} A-k_{1 x} k_{1 y}\right] .
$$

Again, the dependence of $\phi_{12}\left(k_{x}, y\right)$ on $\Omega(y)$ is universal, $\phi_{12}\left(k_{x}, y\right) \propto 1 / \Omega$, which implies $\phi_{12}\left(k_{x}, y\right) \propto y$ in the $\log$ range. Dependence on $k_{x}$ is universal for $k_{x} \ll k_{*}$,

$$
\phi_{12}=\frac{c_{12}}{\Omega k_{x}^{2}},
$$

where the constant $c_{12}$ is

$$
\begin{aligned}
c_{12}= & \frac{1}{(2 \pi)^{3}} \int_{0}^{\infty} \mathrm{d} \beta \int_{-\infty}^{\infty} \mathrm{d} k_{1 y} \int_{-\infty}^{\infty} \mathrm{d} k_{1 z} \frac{3 F\left(0, k_{1 y}, k_{1 z}\right)}{8 \pi} \frac{\left(k_{1 y}^{2}+k_{1 z}^{2}\right)^{3}}{\left(\left(k_{1 y}+\beta\right)^{2}+k_{1 z}^{2}\right) k_{1 z}} \\
& \times\left(\arctan \frac{k_{1 z}}{k_{1 y}+\beta}-\arctan \frac{k_{1 z}}{k_{1 y}}\right) .
\end{aligned}
$$


To find $\phi_{22}$ we note that viscosity is unimportant and can be set equal to zero in this case too. We have

$$
\phi_{22}=\frac{1}{(2 \pi)^{2} \Omega k_{x}} \int_{0}^{\infty} \mathrm{d} \beta \int_{R^{2}} \mathrm{~d} k_{1 y} \mathrm{~d} k_{1 z} \frac{3 F\left(\boldsymbol{k}_{1}\right)}{8 \pi} \frac{k_{1}^{4}}{\left(\left(k_{1 y}+\beta\right)^{2}+k_{h}^{2}\right)^{2}} k_{h}^{2} \frac{1}{4 \pi \Omega k_{x}} \int_{R^{2}} \mathrm{~d} k_{1 y} \mathrm{~d} k_{1 z} \frac{3 F\left(\boldsymbol{k}_{1}\right)}{8 \pi} \frac{k_{1}^{4}}{k_{h}},
$$

so that

$$
\phi_{22} \rightarrow \frac{c_{22}}{\Omega k_{x}} \text { for } k_{x} / k_{*} \rightarrow 0,
$$

where

$$
c_{22}=\frac{1}{4 \pi} \int_{R^{2}} \mathrm{~d} k_{1 y} \mathrm{~d} k_{1 z} \frac{3 F\left(0, k_{1 y}, k_{1 z}\right)}{8 \pi} \frac{\left(k_{1 y}^{2}+k_{1 z}^{2}\right)^{4}}{\left|k_{1 z}\right|}=\text { const. }
$$

Finding $\phi_{33}$ is similar to finding $\phi_{11}$ in that the asymptotic expression for $v \rightarrow 0$ is dominated by large $\beta$ 's. We find

$$
\phi_{33}=\frac{c_{33}}{v^{1 / 3} \Omega^{2 / 3} k_{x}^{2 / 3}},
$$

where $c_{33}$ tends to a constant in the limit $k_{x} / k_{*} \rightarrow 0$,

$$
c_{33}=\left.\frac{3}{4(2 \pi)^{3}}\left(\int_{0}^{\infty} \mathrm{e}^{-\left(2 p^{3} / 3\right)} \mathrm{d} p\right) \int_{R^{2}} \mathrm{~d} k_{1 y} \mathrm{~d} k_{1 z} F\left(0, k_{1 y}, k_{1 z}\right)\left(k_{1 y}^{2}+k_{1 z}^{2} \kappa_{32}^{2}-k_{1 y} k_{1 z} \kappa_{32}\right)\right|_{k_{x}=0} .
$$

The remaining two components of the spectrum tensor, $\phi_{13}$ and $\phi_{23}$ are equal to 0 when $F\left(k_{x}, k_{y},-k_{z}\right)=$ $F\left(k_{x}, k_{y}, k_{z}\right)$.

\section{Conclusion}

In this paper we presented a rigorous analytical theory of shear-flow turbulence forced by a weak small-scale external forcing. The weakness and small-scale of the forcing allowed us to use scale-separation and RDT. The model was closed by combining RDT for turbulence with the Reynolds-averaged equations for the mean flow. Such an analytical theory is designed to provide exact reference solutions against which more general but less rigorous phenomenological theories could be tested. In real shear-flow turbulence (e.g. near-wall turbulence) the turbulence forcing outside of the viscous sublayer is provided by the continuous supply of vorticity propagating into the outer regions as a result of intermittent bursts of coherent vortices generated inside the viscous sublayer. In our formulation we decouple the turbulence description from such strongly nonlinear dynamics by considering the turbulence forcing to be external. It is this decoupling that allows us to obtain rigorous analytical solutions and study their dependence on the properties of the initial vorticity.

We found the conditions on the forcing under which one may expect the log-law solution for the mean velocity profile. This happens if, in addition to being weak and small-scale, the initial vorticity is shortly correlated in time and statistically uniform in space. Thus, we conclude that the mean profile is very sensitive to the properties of the initial vorticity. For example, if the second correlator of the forcing is not constant, but is a power-law function of the distance from the wall, then the mean profile is a power-law rather than the log-law. In particular, it can be of the scaling form proposed by Barenblatt et al. [4-6]. Such a dependence of the forcing on the distance from the wall can be viewed as incomplete mixing of the initial vorticity material (resulting from the sublayer bursts) over the overlap region. If not well mixed, the initial vorticity is intermittent (i.e. not space filling). Barenblatt et al. suggested that vorticity intermittence may be responsible for the violation of complete similarity in the overlap region which, in turn, can lead to 
the power-law mean velocity profiles [4-6]. Our results agree with this view and, moreover, we identify those statistical properties of the initial vorticity which are essential for complete similarly and the log-law. Our results suggest that these properties, i.e. the spatial uniformity and fast decay of the second correlator, should be examined using DNS data. This can be done by tracking the vorticity generated in the viscous sublayer by marking it with passive particles.

It is interesting that the experimental data Zagarola et al. [7,8] obtained for high-pressure pipe air flow suggest the existence of two overlap regions, one of which has a power-law profile (for $50<y_{+}<500$ ) and another having a logarithmic region (at $500<y_{+}$and $y<0.1 \mathrm{H}$ ), where we have a power-law. It is therefore possible that the initial vorticity is distributed differently over these two regions.

Our analytical results give interesting information about the role of viscosity outside of the viscous sublayer. Recall that according to classical complete similarity viscosity has no effect on the averaged quantities in this region. In contrast, we see that the main contribution to the turbulent shear stress, $\tau_{\mathfrak{c}}$, has a very strong dependence on viscosity in this region: it is inversely proportional to $v$ for small $v$. However, if the initial vorticity is uniform in space then $\tau_{\mathrm{c}}$ is independent of $y$ and, therefore, it does not contribute to the mean flow dynamics. On the other hand, the part of the turbulent shear stress $\tau$ that does contribute to the mean flow dynamics can be either independent of $v$ (for symmetric forcing), or logarithmically dependent on $v$ if the forcing is asymmetric. Dependence of the turbulent stress on viscosity is not surprising given that according to RDT turbulence energy is growing because of vortex stretching by the mean shear, and it is the viscous cut-off that determines the maximum turbulence amplitude. As we saw, however, such a viscosity dependence does not preclude existence of the log profile. Note also that the main contribution to $\tau_{\mathrm{c}}$ and $\tau_{\mathrm{a}}$ comes from very elongated streaks with $k_{y}, k_{z} \gg k_{x}$ whereas $\tau_{\mathrm{s}}$ is mostly generated by isotropic scales, $k_{y} \sim k_{z} \sim k_{x}$.

Note that some of the assumptions made in the present paper about the turbulence forcing (i.e. its uniformity and short correlation time) are necessary for the log solution to exist, but they not important for the applicability of the approach developed here. On the other hand, such assumptions as the weakness and small-scale of the turbulence forcing are essential for the validity of RDT and the scale separation technique. In the introduction we presented an intuitive argument for the assumption that the initial vorticity can be small-scale and weak, but a reliable conclusion about validity of these assumptions (and the distances from the wall for which they are valid) can be obtained only by further detailed experimental and numerical analysis of the vorticity field.

It is interesting that, as Kevlahan and Hunt [26] pointed out, RDT may be a valid approach even if the maximum vorticity perturbation in turbulence is greater than the mean shear. Indeed, the maximum vorticity usually lies in very small-scale perturbations which contain negligible energy and which do not contribute to the shear stress. In other words, the vorticity field is "contaminated" by the very small scales that are strongly nonlinear (and have $-5 / 3$ energy spectrum), but they may be ignored as they do not feedback on the mean flow. Thus, it is only important that the vorticity of the energy containing scales is smaller than the mean shear which is a much milder restriction on validity of RDT. A similar argument applies to the scale separation: only the energy containing turbulent scales need be separated from the mean flow scale.

The importance of the exact results obtained in the present paper is that they form the basis of a more general, but less rigorous, model. Such a model is defined by a self-consistent choice of the turbulence forcing in such a way that, if possible, the theory reproduces all the experimental dependencies measured to date (such as the mean profiles, etc.). A model of this type is described in a separate publication [22] where we also show how to include turbulent scales which are not well separated from the mean flow scale. Another, and perhaps more consistent, way to develop such a model would be to measure the properties of the starting vorticity (and, therefore, turbulence forcing) directly by tracking propagation of the sublayer generated vorticity into the overlap region. This is a challenging task for laboratory experiment, but it is a tractable and well-defined problem to be tackled by DNS. Ideally, the future theory of near-wall turbulence should include a model for the generation of the viscous sublayer vortices and 
their propagation into the overlap region, and, therefore, constitute a closed system for the turbulent region coupled to the viscous sublayer dynamics.

\section{Acknowledgements}

We thank Vladimir Lebedev for helpful discussions. N. Kevlahan gratefully acknowledges support from McMaster University.

\section{References}

[1] S.V. Nazarenko, N. Kevlahan, B. Dubrulle, J. Fluid Mech. 390 (1999) 325.

[2] Th. von Karman, Mechanische Aehnlichkeit und Turbulenz, Nach. Ges. Wiss. Goettingen Math-Phys. Klasse, 1932 , pp. 58-76.

[3] L. Prandtl, Zur turbulenten Stroemung in Rohren und laengs Platten, Ergeb. Aerodyn. Versuch., Series 4, Goettingen, 1932.

[4] G.I. Barenblatt, J. Fluid Mech. 248 (1993) 513.

[5] G.I. Barenblatt, A.J. Chorin, Proc. Nat. Acad. Sci. USA 93 (1996) 6749.

[6] G.I. Barenblatt, A.J. Chorin, V.M. Prostokishin, Proc. Nat. Acad. Sci. USA 94 (1997) 773.

[7] M.V. Zagarola, A.J. Smits, Phys. Rev. Lett. 78 (1997) 239.

[8] M.V. Zagarola, A.E. Perry, A.J. Smits, Phys. fluids 9 (1997) 2094.

[9] M. Oberlack, J. Fluid Mech. 379 (1999) 1.

[10] W. George, L. Castillo, M. Wosnik, TAM Report No. 872, UILU-ENG-97-6033, University of Illinois at Urbana-Champain, IL, November 1997.

[11] J. Kim, R. Moser, J. Fluid Mech. 177 (1987) 133.

[12] R. Moser, Phys. Fluids, April (1999) 943.

[13] H.K. Moffatt, The interaction of turbulence with a strong wind shear, in: A.M. Yaglom, V.I. Tatarsky (Eds.), Proceedings of the URSI-IUGG International Colloquim on Atmospheric Turbulence and Radio Wave Propagation held in Moscow, June 1965, Nauka, Moscow, 1967.

[14] G.K. Batchelor, I. Proudman, Q. J. Mech. Appl. Math. 7 (1954) 83.

[15] J.C.R. Hunt, J. Fluid Mech. 61 (1973) 625.

[16] A. Lifschitz, E. Hameiri, Phys. Fluids A 3 (1991) 2644.

[17] J. Nikuradze, Forsch. Arb. Ing-Wes No. 356, 1932 (English translation NACA TT F-10, p. 359).

[18] A.I. Dyachenko, S.V. Nazarenko, V.E. Zakharov, Phys. Lett. A 165 (1992) 330.

[19] B. Dubrulle, S. Nazarenko, Physica D 110 (1997) 123.

[20] D.Yu. Manin, S.V. Nazarenko, Phys. Fluids A 6 (1994) 1158.

[21] M.T. Landahl, Physica D 37 (1989) 11.

[22] B. Dubrulle, J.-P. Laval, S. Nazarenko, N.K.-R. Kevlahan, Derivation of equilibrium profiles in plane parallel flows using a dynamic subgrid-scale model, submitted to Phys. Fluids.

[23] S. Nazarenko, Phys. Lett. A 264 (2000) 444.

[24] I.S. Gradshtein, I.M. Ryzhik, Table of Integrals, Series, and Products, Academic Press, New York, 1965.

[25] A.E. Perry, S. Henbest, M.S. Chong, J. Fluid Mech. 165 (1986) 163.

[26] N.K.-R. Kevlahan, J.C.R. Hunt, J. Fluid Mech. 337 (1997) 333. 